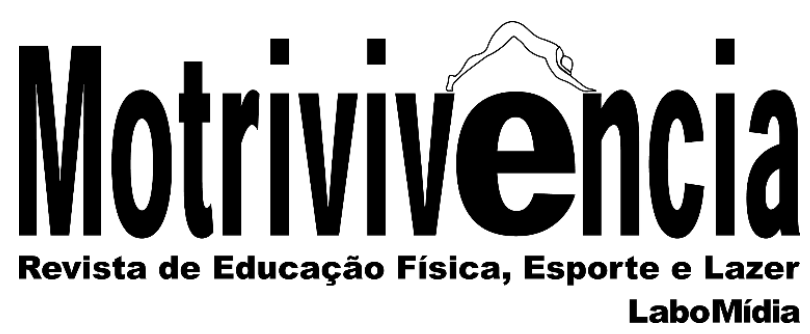

\title{
Centro de estudos em política e gestão do esporte e lazer: trajetória científica e perspectivas futuras
}

\section{RESUMO}

O Centro de Estudos em Política e Gestão do Esporte e Lazer se apresenta como um grupo de pesquisa vinculado a Universidade Estadual do Sudoeste da Bahia. A referida entidade tem como prerrogativa o desenvolvimento de pesquisas correlatas ao processo de formação da agenda para o Esporte, Lazer, Promoção da Saúde e Educação Física. Do ponto de vista epistemológico, o programa de pesquisa do referido grupo encontra-se alicerçado na Ciência Política. A produção do conhecimento encontra-se materializada em livros, capítulos de livros e artigos publicados em periódicos nacionais e internacionais. Diante do exposto, os estudos desenvolvidos pelo grupo tornam-se uma alternativa para a revisão teórica da literatura científica nacional com vistas ao processo de falseamento dos valores e crenças que permeiam o fazer científico da produção relacionada a política e gestão do esporte e lazer.

PALAVRAS-CHAVE: Política; Gestão; Esporte; Lazer; Promoção da saúde
Neidiana Braga da Silva Souza

Mestra Universidade Estadual de Maringá,

Departamento de Educação Física, Maringá, Paraná, Brasil. neidianabraga@gmail.com

근 https://orcid.org/0000-0002-0480-0430

Temistocles Damasceno Silva Mestre Universidade Estadual do Sudoeste da Bahia, Departamento de Saúde I, Jequié, Bahia, Brasil. tom@uesb.edu.br

https://orcid.org/0000-0001-5932-9773

Ivan Luiz Ferreira da Silva Graduado Universidade Estadual do Sudoeste da Bahia,

Departamento de Saúde I, Jequié, Bahia, Brasil. ivanluizfs@gmail.com

ำ https://orcid.org/0000-0002-6122-9912

Murillo Lago Menezes Especialista Universidade Estadual de Maringá, Departamento de Educação Física,

Maringá, Paraná, Brasil. murillolago_@hotmail.com (ํ) https://orcid.org/0000-0002-8629-1325 
The center for studies in sports and leisure policy and management: scientific trajectory and future perspectives

\begin{abstract}
The Center for Studies in Sports and Leisure Policy and Management presents itself as a research group linked to the State University of Southwest Bahia. This entity aims to develop research related to the process of forming the agenda for Sport, Leisure, Health Promotion and Physical Education. From an epistemological point of view, the research program of that group is based on Political Science. The production of knowledge is materialized in books, book chapters and articles published in national and international journals. Given the above, the studies developed by the group become an alternative for the theoretical review of the national scientific literature with a view to the process of falsifying the values and beliefs that permeate the scientific production of production related to the politics and management of sport and leisure.
\end{abstract}

KEYWORDS: Politics; Management; Sport; Recreation; Health promotion

\title{
El centro de estudios en política y gestión del deporte y el ocio: trayectoria científica y perspectivas de futuro
}

\section{RESUMEN}

El Centro de Estudios en Política y Gestión del Deporte y el Ocio se presenta como un grupo de investigación vinculado a la Universidad Estatal del Sudoeste de Bahía. Esta entidad tiene como objetivo desarrollar investigaciones relacionadas con el proceso de conformación de la agenda de Deporte, Ocio, Promoción de la Salud y Educación Física. Desde un punto de vista epistemológico, el programa de investigación de ese grupo se basa en la Ciencia Política. La producción de conocimiento se materializa en libros, capítulos de libros y artículos publicados en revistas nacionales e internacionales. Dado lo anterior, los estudios desarrollados por el grupo se convierten en una alternativa para la revisión teórica de la literatura científica nacional con miras al proceso de falsificación de los valores y creencias que permean la producción científica de producción relacionada con la política y gestión del deporte y el ocio.

PALABRAS-CLAVE: Política; Administración; Deporte; Recreación; Promoción de la salud 


\section{INTRODUÇÃO}

As distintas mudanças sociais que ocorrem constantemente em escala global provocam efeitos colaterais em diversas esferas da sociedade, dentre elas, a ciência. Ao observar o desenvolvimento científico, deve-se considerar que o conhecimento produzido torna-se provisório e, consequentemente, com o avanço dessa nova dinâmica social, suas bases passam a ser questionadas, colocadas à prova frequentemente, com o intuito de produzir cientificamente perspectivas mais próximas da realidade em vigência (POPPER, 1974; 1980; BECK, 2011).

A partir das conjecturas e refutações estabelecidas através dos critérios de testabilidade e falseabilidade de teorias é que acontece o avanço científico. Sendo assim, as bases científicas passam a se (auto) questionarem, reflexo da amplitude das tecnologias e do acesso às informações evidenciadas pela ambiguidade presente na modernidade (GIDDENS, 1996; BECK,1997). Atrelado a esta perspectiva de transformações sociais, percebe-se que existem interligações globalizadas que influem no jogo científico. Bunge (1980) afirma que tal feito é intensificado pelas redes/subsistemas interligados nacionalmente e internacionalmente, que atuam no estabelecimento de critérios científicos e no desenvolvimento de teorias, através do aumento quantitativo e qualitativo das produções.

Para tal, Morin (2006) concebe a importância do pensamento complexo na ciência como um meio de identificação da complexidade da realidade, permitindo uma análise macro e microdimensional e reduzindo as possibilidades de equívocos, expressos na patologia do saber dogmatismo teórico. Por tanto, não se pode ignorar que o processo de (auto) questionamento das bases, contribuem para a falseabilidade das teorias, favorecendo o progresso científico de forma conjectural.

A inter-relação entre leigos e especialistas neste jogo, conforme Beck (1997), deixam em destaque que o acúmulo de "certezas" através da especialização, provocam incertezas e provisoriedade dos conhecimentos produzidos em autorreflexão. Nesta lógica, o papel dos especialistas em busca de progresso científico se localiza como peça chave na destituição da patologia do saber. Para Bourdieu (1968), aqueles que buscam o rompimento com os dogmas, acabam criando espaços subversivos, com vistas a objetivação do sujeito objetivante ${ }^{1}$.

Neste exercício de (auto) questionamento científico e da busca por vigilância epistemológica, o Centro de Estudos em Política e Gestão do Esporte e Lazer (CEGEL/UESB) almeja estabelecer uma relação entre as estruturas narrativas com a ação dos atores que compõem a definição dos problemas e alternativas com vistas a compreensão das diversas variáveis que

\footnotetext{
1 De acordo com Bourdieu (2004), vigilância epistemolôgica se refere ao "afastamento" dos sujeitos (seus valores e
} crenças) para evitar uma análise enviesada do objeto investigado. 
influenciam as políticas públicas de esporte, lazer, promoção da saúde e Educação Física. Para tal, o referido grupo propõe uma agenda de pesquisa pautada nos pressupostos teóricos que permeiam o processo de formação das agendas política e governamental.

\section{A formação da agenda como ferramenta de análise da política pública de esporte e lazer}

Ao considerar que a atenção é um recurso escasso e que tanto pessoas quanto organizações estabelecem prioridades de atuação, estudar a formação da agenda significa identificar como o governo distribui atenção entre as diversas questões existentes, decidindo o que é um problema a ser priorizado (BAUMGARTNER; JONES, 2015). O ponto central dos estudos sobre agenda perpassa pela compreensão sobre a forma como alguns problemas são considerados mais importantes do que outros em um determinado momento, mobilizando a ação governamental (ZAHARIADIS, 2016).

As análises sobre formação de agenda foram iniciadas na década de 1970 por meio dos estudos desenvolvidos por Cobb e Elder $(1971 ; 1972)$, no intuito de compreender de que forma as questões são criadas e como se processa a formação da agenda. Na década de 1980, John Kingdon elaborou o modelo de múltiplos fluxos para compreender como uma questão pode ser inserida na agenda. Tal modelo parte da premissa que a ação governamental sofre influência de três fluxos (problemas, alternativas e políticas). Neste quesito, as mudanças na agenda estão condicionadas ao surgimento de janelas de oportunidade durante o período de convergência dos fluxos abordados. Logo, torna-se necessário compreender de que forma esses fluxos se relacionam e em que medida afetam o processo de formulação da política.

Para Kingdon (2003, p.165), a convergência dos fluxos de problemas, soluções e políticos, torna-se fundamental para a constituição daquilo que o autor denomina janelas de oportunidade política (policy windows). Esse processo se materializa a partir do momento em que o fluxo de problemas despertam a atenção do governo e encontram o ambiente político favorável para ascensão a agenda. Para o autor, apesar do fluxo de soluções não intervir diretamente na formação da agenda é imprescindível para o processo de transição das questões que encontram-se na agenda governamental migrarem para a agenda decisória. Capela $(2018$, p.43) ressalta que as janelas de oportunidade política podem se manifestar de maneira previsível ou imprevisível. As alterações político-ideológicas ocorridas no governo por meio das eleições caracterizam as situações previsíveis enquanto as imprevisíveis podem ser representadas por crises oriundas de eventos inesperados que também ocasionam mudanças na agenda.

Ademais, o modelo do Equilíbrio Pontuado elaborado por Baumgartner e Jones em 1993 possibilitou análises das continuidades e mudanças na agenda governamental. $\mathrm{O}$ modelo foi 
apresentado como ferramenta de compreensão da mudança política correlata aos longos períodos de estabilidade que sofrem alterações pontuais em relação ao cotidiano político. Vale destacar que os estudos desenvolvidos por Kingdon sobre a formação da agenda foram essenciais para a elaboração dos conceitos que permeiam o modelo em questão. A racionalidade limitada, os processos baseados na incerteza e as informações imperfeitas foram elementos absolvidos na constituição do modelo. Contudo, Baumgartner e Jones (1993) propuseram um avanço significativo sobre os pressupostos de Kingdon, ao inserir a influência dos arranjos institucionais na explicação da referida dinâmica. Sendo assim, os atores, as ideias e os arranjos institucionais ${ }^{2}$, se apresentam como elementos centrais para a compreensão da atenção dada a determinada questão e as prioridades elencadas no processo de formação de agenda.

A utilização desses modelos teóricos tornou-se recorrente na literatura científica nacional. Vale ressaltar que até a década de 1980 os estudos sobre política pública no Brasil estavam relacionados ao desenvolvimento de análises macrossociológicas acerca do Estado e compreensão das consequências do processo político nas várias esferas que constituem a sociedade brasileira. Os anos subsequentes tornaram-se um marco temporal de composição da literatura especializada em políticas públicas por meio de estudos delineados em diversas disciplinas (MARQUES; FARIA, 2018).

Apesar da recorrência histórica de pesquisas sobre a relação estabelecida entre a política e o esporte nos estados brasileiros, a análise sobre a formação da agenda para o esporte, o lazer, a promoção da saúde e a Educação Física ainda se apresenta de maneira embrionária, tornando-se assim, um desafio para os pesquisadores da área. Para Mendes (2017, p. 2), "os estudos em proximidade com este tema no Brasil simplesmente apresentam uma descrição da legislação e da estrutura institucional voltada à execução da política de esporte no país”. As revisões sistemáticas elaboradas por Castro et al. (2012) e Amaral et al. (2014), indicam que a maior parte do conhecimento científico em políticas públicas de esporte produzido pela área de Educação Física no Brasil ignora os objetos de pesquisa em políticas públicas, tais como: as instituições, as regras, os grupos de interesse, a formulação da política e o processo decisório.

Ao considerar a formação da agenda enquanto elemento central do processo de produção de políticas públicas (KINGDON, 2003, p.3) e ferramenta crucial para a compreensão das prioridades elencadas por parte do governo (BAUMGARTNER; JONES, 2015) constata-se uma possibilidade

\footnotetext{
${ }^{2}$ Os atores constituem as tomadas de decisão e ao mesmo tempo, estabelecem disputas de poder na arena decisória (instituições), sendo motivados por interesses particulares ou conduzidos por fatores externos. As ideias se apresentam como valores políticos que revestem os posicionamentos e justificam as ações do poder público. Os arranjos institucionais são ações desenvolvidas pelos atores em relação as diversas variáveis que influenciam o processo de formulação da política (BAUMGARTNER; JONES, 1993). Vale ressaltar que esses conceitos serão explorados amplamente no capítulo ll, o qual diz respeito ao referencial teórico de análise da pesquisa.
} 
de compreender a atenção dada ao esporte, lazer, promoção da saúde e Educação Física no contexto dos estados brasileiros bem como nos países da América Latina. Logo, os estudos sobre a formação da agenda podem colaborar para a compreensão em torno das prioridades estabelecidas pelos partidos políticos no que diz respeito aos fenômenos abordados e ao mesmo tempo, contribuir para o entendimento acerca das mudanças ocorridas no campo político e a influência das ideais, atores e arranjos, no processo de constituição da agenda.

\section{O CEGEL e a produção do conhecimento sobre política e gestão do esporte e lazer}

O CEGEL surgiu em meados de 2012 enquanto espaço de produção do conhecimento científico na área de política e gestão do esporte e lazer. O grupo foi idealizado por professores e discentes da Universidade Estadual do Sudoeste da Bahia - UESB. A principal premissa relacionava-se ao processo de elaboração de evidências científicas com vistas ao auxílio da tomada de decisão na gestão pública municipal.

A produção do conhecimento delineada pelo referido grupo de pesquisa, historicamente, foi compartilhada com o campo científico a partir do uso de diferentes mecanismos de publicização de estudos. Neste sentido, utilizou-se de livros, capítulos de livros, artigos e outras produções técnicas. Tal premissa se materializou na publicação de obras como o livro POLÍTICAS PÚBLICAS DE ESPORTE NO INTERIOR DA BAHIA, publicado em 2017, o qual teve em seu escopo a participação de três universidades estaduais baianas, a saber: Universidade do Estado da Bahia (UNEB), Universidade Estadual de Feira de Santana (UEFS) e Universidade Estadual do Sudoeste da Bahia (UESB). Tais entidades de ensino foram representadas por quatro grupos de pesquisa, dentre eles, o Centro de Estudos em Política e Gestão do Esporte e Lazer (GEGEL).

O respectivo livro reuniu diversas pesquisas acerca das Políticas Públicas de Esporte e Lazer em municípios do interior da Bahia no intuito de compreender a dinâmica política correlata ao esporte com base em diferentes elementos, tais como: legislação; infraestrutura; investimento público; entre outros. Destaca-se que a obra supracitada corresponde ao volume I da Série Política e Gestão de Esporte e Lazer. O volume II desta série, intitulado de POLÍTICAS PÚBLICAS DE LAZER NO INTERIOR DA BAHIA, publicado em 2017, também contou com a participação do CEGEL em seu desenvolvimento. Neste contexto, o livro buscou compreender em que medida os municípios baianos desenvolveram Políticas Públicas de Lazer para os cidadãos, estabelecendo relações entre as políticas desenvolvidas no âmbito municipal e o contexto brasileiro.

No ano de 2019, O CEGEL participou da organização do livro O ESPORTE NAS ELEIÇÕES 2018. A obra apresenta uma compilação das propostas para o esporte elencadas nos 
planos de governo correspondentes as candidaturas à Presidência da República e ao Governo dos estados e do Distrito Federal. Trata-se de um marco temporal para os estudos sobre a atenção dada ao esporte na arena política durante o processo eleitoral. A obra foi construída em parceria com pesquisadores membros do Grupo de Estudos e Pesquisa em Políticas Públicas de Esporte e Lazer (GEPPOL) da Universidade Estadual de Maringá (UEM) e da Secretaria Estadual Paraná do Colégio Brasileiro de Ciências do Esporte (CBCE-PR).

Em 2020, o grupo participou da organização do livro "EDUCAÇÃO FÍSICA: uma abordagem multidisciplinar". Trata-se de uma iniciativa da Associação de Professores e Profissionais da Instituições de Ensino Superior em Educação Física da Bahia (APIESEF) que contou com a participação de pesquisadores vinculados ao CEGEL. No mesmo ano, o CEGEL publicou o livro "Políticas Públicas de Esporte e Lazer: sistemas nacionais e políticas municipais", o qual aborda as políticas públicas de esporte e lazer com um olhar direcionado a diferentes entes federativos, sociedade civil e coalizões de governos. A obra contou com a participação de mais de 30 autores que abordaram questões diversas, tornando o trabalho mais amplo. Neste sentido, podese destacar questões como: gestão pública municipal; envolvimento de prefeituras com o esporte profissional; intersetorialidade; descentralização e financiamento do esporte e lazer.

Além disso, o grupo apresenta um número significativo de trabalhos correlatos ao processo de compreensão da atenção dada aos fenômenos supracitados nas agendas política e governamental. Tais estudos tiveram autoria de pesquisadores do CEGEL e foram publicados em diversos livros relacionados as áreas de Educação Física e de Políticas de Esporte e Lazer. Nesta lógica, vários artigos foram publicados em periódicos nacionais e internacionais. Além disso, inaugurou-se a agenda de pesquisa sobre a promoção da saúde com base na publicação de um artigo científico acerca da atenção dada ao referido tema nas agendas política e governamental dos estados brasileiros.

No âmbito das ações extensionistas, o grupo organizou um curso de capacitação para os gestores esportivos que atuavam na gestão pública dos municípios baianos. Outra ação que merece destaque refere-se a organização do I Congresso Baiano de Política e Gestão do Esporte e Lazer no ano de 2019. Além disso, o grupo foi responsável pela organização do III Seminário de Políticas Públicas de Esporte e Lazer em parceria com o Grupo de Estudos e Pesquisas em Políticas de Esporte e Lazer (GEPPOL-UEM) bem como o Pré-Enarel Região Nordeste em parceria com o Grupo de Estudos do Lazer (GEL-UEM).

Concomitantemente, o grupo participou de diversas ações com o objetivo de auxiliar o processo de planejamento e avaliação da política e gestão do esporte e lazer por meio da realização de audiências públicas, seminários, fóruns e conferências realizadas em parcerias com diversos 
municípios do estado da Bahia, dentre eles: Jequié; Ipiaú; Jaguaquara; Ibirataia; Santa Cruz Cabrália; Itaquara; Itacaré; Ituberá; Belo Campo; Caetanos; Santa Inês. Tais ações, visam contribuir, com a construção de alternativas viáveis para o fomento das políticas públicas de esporte e lazer com base em evidências científicas.

Atualmente, o grupo trabalha no desenvolvimento de um manual e um software de gestão esportiva voltado ao assessoramento dos profissionais que atuam na referida área. Neste contexto, o processo de desenvolvimento do programa relaciona-se a associação dos indicadores socioeconômicos ao processo de formulação das ações a serem implementadas. Além disso, o programa pretende correlacionar os programas e projetos delineados pelas unidades administrativas com o aparato legal do setor e com as demandas elencadas através dos mecanismos de gestão participativa. Na sequência, almeja-se utilizar o programa para o monitoramento da alocação e execução financeira destinada a função desporto e lazer e ao mesmo tempo, avaliar os planos de ação voltados a construção e manutenção de espaços e equipamentos esportivos. Sendo assim, acredita-se que o programa possa estabelecer uma aproximação entre a ciência e a política, ação salutar para o desenvolvimento da área.

\section{As possibilidades de desenvolvimento de pesquisas com base na análise do processo de formação da agenda para o esporte, lazer, promoção da saúde e Educação Física.}

Nos últimos anos, o CEGEL propôs uma agenda de pesquisa direcionada a análise da política e gestão do esporte e lazer nos municípios brasileiros, analisando os diversos elementos que compõem a gestão pública municipal. Futuramente, pretende-se ampliar o desenvolvimento de estudos comparativos, no que se refere as unidades federativas brasileiras bem como os países da América Latina. Para tal, espera-se desenvolver estudos transversais e longitudinais, considerandose as variáveis políticas e institucionais relacionadas a agenda.

As pesquisas pretendem detectar as prioridades governamentais para as políticas públicas de esporte, lazer, promoção da saúde e Educação Física nas 27 Unidades federativas brasileiras. Logo, pretende-se estabelecer uma agenda de pesquisa nacional e internacional, com o propósito de realizar uma análise comparativa, em períodos e cenários políticos distintos. Nesse contexto, o grupo fará uso dos pressupostos teóricos e metodológicos da Ciência Política, no intuito de evidenciar a lógica de funcionamento dos processos políticos estatais que envolvem a agenda.

Para Houlihan, Bloyce e Smith (2009), as análises das atividades governamentais direcionadas para o esporte nos últimos 20 anos podem fornecer um grande número de evidências do crescimento no interesse e intervenção do Estado em uma ampla quantidade de países. Nessa 
lógica, o Centro de Estudos em Política e Gestão do Esporte e Lazer intenta-se a investigar os níveis de atenção dos governos nacionais e subnacionais para as temáticas supracitadas.

Ao realizar uma diagnose das ações elaboradas, dos conteúdos nelas inseridos e o período que as questões permaneceram nas agendas, será possível compreender as aproximações e distanciamentos entre tais agendas em diferentes países do mundo. Portanto, é necessário refletir criticamente sobre a natureza da intervenção estatal no esporte, no lazer, na promoção da saúde e na Educação Física, estruturando uma agenda de pesquisa que acompanhe o envolvimento do Poder Público com essas políticas.

Desse modo, será possível examinar o planejamento das ações e estabelecer relações baseadas em uma abordagem comparativa entre os entes federados. Com a finalidade de diagnosticar a configuração político-partidária dos entes federativos brasileiros a serem investigados, almeja-se coletar dados das eleições municipais, estaduais e federais, disponibilizados pelo Tribunal Superior Eleitoral (TSE). Levando em consideração as particularidades políticas inseridas nos cenários geográficos das pesquisas, objetiva-se demonstrar as pautas apresentadas pelas diferentes legendas partidárias nos recortes temporais a serem elencados nas pesquisas. Os documentos públicos serão utilizados como ferramentas substanciais para identificar os problemas e alternativas para as políticas mencionadas na arena política durante o período eleitoral e no ambiente governamental.

Neste sentido, almeja-se compreender e explicar a dinâmica das agendas de modo a elucidar os processos de formulação das políticas, mediante à identificação de evidências consistentes e construção de hipóteses possíveis de explicar as mudanças políticas no decorrer dos anos. Ademais, serão utilizadas lentes teóricas coerentes com o espaço político-governamental, propostas pela Ciência Política, as quais serão testadas empiricamente por meio de uma perspectiva falseacionista.

Nesta lógica, torna-se necessária a realização de análises empíricas e consequente produção teórica sobre tais fenômenos, tendo em vista que a lacuna existente em torno dos estudos sobre a formação da agenda dessas políticas se apresenta como elemento pertinente para a ampliação da discussão no âmbito acadêmico. Os estudos a serem realizados na literatura científíca precisam compreender os níveis de envolvimento dos governos nas políticas públicas com vistas ao acompanhamento do planejamento e do processo político-institucional correlatos aos temas supracitados. 


\section{REFERÊNCIAS}

AMARAL, Silvia Cristina Franco; RIBEIRO, Olívia Cristina Ferreira; SILVA, Dirceu Santos. Produção científico-acadêmica em Políticas Públicas de Esporte e Lazer no Brasil. Motrivivência, v. 26, n. 42, p. 27-40, 2014.

BAUMGARTNER, Frank R.; JONES, Bryan D. Agendas and instability in American politics. University of Chicago Press, 1993.

BECK, Ulrich. Sociedade de risco: rumo a uma outra modernidade. São Paulo: Editora 34. $2^{\mathrm{a}}$ ed. 2011.

BECK, Ulrich. A reinvenção da política: rumo a uma teoria da modernização. BECK, U.;

GIDDENS, A.; LASH, S. Modernização reflexiva. São Paulo: Ed. da Unesp, 1997.

BOURDIEU, Pierre. Os usos sociais da ciência. São Paulo: Editora Unesp, 2004.

BUNGE, Mario Augusto. Epistemologia: curso de atualização. São Paulo: Editora da USP, 1980.

CAPELLA, Ana Cláudia Niedhardt. Estudos sobre formação da agenda de políticas públicas: um panorama das pesquisas no Brasil. Revista de Administração Pública, Rio de Janeiro, v. 54, n. 6, p. 1498-1512, Dec. 2020.

CAPELLA, Ana Cláudia Niedhardt. Formulação de Políticas. Brasília: Enap, 2018.

CASTRO, Suélen Barboza Eiras de; MORO, Neuza Rosa Nery de Lima.; SILVEIRA, Liliane Roberta Tsumanuma da; MEZZADRI, Fernando Marinho. O estado da arte em políticas sociais de esporte e lazer no Brasil (2000-2009). Pensar a Prática, 15(2), 2012.

COBB, Roger W.; ELDER, Charles D. Participation in american politics: the dynamics of agenda building. Boston: Allyn and Bancon, 1972.

COBB, Roger W.; ELDER, Charles D. The politics of agenda-building: an alternative perspective for Modern Democratic Theory. Journal of Politics, v. 33, n. 4, p. 892-915, 1971.

GIDDENS, Anthony. Para além da esquerda e da direita. São Paulo: Unesp. 1996.

HOULIHAN, Barrie; BLOYCE, Daniel; SMITH, Andy. Developing the research agenda in sport policy. International Journal of Sport Policy and Politics, London, 2009.

KINGDON, John W. Agendas, alternatives, and public policies. 3a. ed. New York: Harper Collins, 2003.

MENDES, Alessandra Dias. Política da política de esporte: uma análise da Frente Parlamentar do Esporte e da Comissão do Esporte da Câmara dos Deputados na $54^{\mathrm{a}}$ Legislatura. Tese (doutorado) - Universidade Federal do Paraná, Curitiba, 2017.

MORIN, Edgar. Introdução ao pensamento complexo. Porto Alegre: Sulina, 2006.

PARSONS, Wayne. Políticas públicas: una introducción a la teoría y la práctica del análisis de políticas públicas. México, Flacso, 2007. 
PETERS, B. Guy. Advanced Introduction to Public Policy. Cheltenham, Northhampton: Edward Elgar, 2015.

POPPER, Karl Raimund. A lógica da pesquisa científica. São Paulo: Cultrix, 1974.

POPPER, Karl Raimund. Conjecturas e Refutações. Brasília: Editora da UnB, 1980.

RITTEL, Horst WJ; WEBBER, Melvin M. Dilemmas in a General Theory of Planning. Policy

Sciences, v. 4, p. 155-169, 1973.

SCHATTSCHNEIDER, Elmer Eric. The Semi-Sovereign People. New York: Winston, 1960.

STONE, Deborah. Policy paradox: the art of political decision making. Nova York: Norton \& Company, [1988], 2002.

ZAHARIADIS, Nikolaos. The domestication of a wild' problem: taming policy agenda setting. In: Zahariadis, N. (org.). Handbook of public policy agenda setting. Cheltenham, Northapton: Edward Elgar, 2016.

\section{NOTAS DE AUTOR}

\section{AGRADECIMENTOS}

Agradecemos aos pesquisadores que contribuíram de maneira efetiva para o desenvolvimento do programa de pesquisa do CEGEL. Ao mesmo tempo, reconhecemos a fundamental importância da Universidade Estadual do Sudoeste da Bahia no processo de materialização das ações planejadas.

\section{CONTRIBUIÇÃO DE AUTORIA}

Não se aplica.

\section{FINANCIAMENTO}

O presente trabalho foi realizado com apoio da Universidade Estadual do Sudoeste da Bahia UESB, Campus de Jequié/BA.

\section{CONSENTIMENTO DE USO DE IMAGEM}

Não se aplica.

\section{APROVAÇÃO DE COMITÊ DE ÉTICA EM PESQUISA}

Não se aplica.

\section{CONFLITO DE INTERESSES}

Não se aplica.

\section{LICENÇA DE USO}

Os autores cedem à Motrivivência - ISSN 2175-8042 os direitos exclusivos de primeira publicação, com o trabalho simultaneamente licenciado sob a Licença Creative Commons Attribution Non-Comercial ShareAlike (CC BY-NC SA) 4.0 International. Esta licença permite que terceiros remixem, adaptem e criem a partir do trabalho publicado, desde que para fins não comerciais, atribuindo o devido crédito de autoria e publicação inicial neste periódico desde que 
adotem a mesma licença, compartilhar igual. Os autores têm autorização para assumir contratos adicionais separadamente, para distribuição não exclusiva da versão do trabalho publicada neste periódico (ex.: publicar em repositório institucional, em site pessoal, publicar uma tradução, ou como capítulo de livro), com reconhecimento de autoria e publicação inicial neste periódico, desde que para fins não comerciais e compartilhar com a mesma licença.

\section{PUBLISHER}

Universidade Federal de Santa Catarina. Programa de Pós-Graduação em Educação Física. LaboMídia - Laboratório e Observatório da Mídia Esportiva. Publicado no Portal de Periódicos UFSC. As ideias expressadas neste artigo são de responsabilidade de seus autores, não representando, necessariamente, a opinião dos editores ou da universidade.

\section{EDITORES}

Mauricio Roberto da Silva, Giovani De Lorenzi Pires, Rogério Santos Pereira.

\section{EDITORES ASSOCIADOS DA SEÇÃO TEMÁTICA}

Fernando Henrique Carneiro; Marcelo de Paula Melo

\section{REVISÃO DO MANUSCRITO E METADADOS}

João Caetano Prates Rocha; Keli Barreto.

\section{HISTÓRICO}

Recebido em: 11 de dezembro de 2020.

Aprovado em: 06 de abril de 2021. 